\title{
Condições socioeconômicas e câncer de cabeça e pescoço: uma revisão sistemática de literatura
}

\author{
Socioeconomic conditions and head and neck cancer: \\ a systematic literature review
}

Antonio Fernando Boing ${ }^{1}$

José Leopoldo Ferreira Antunes ${ }^{2}$

${ }^{1}$ Programa de PósGraduação em Saúde Coletiva, Universidade Federal de Santa Catarina. Campus Universitário. 88040-970 Florianópolis SC.boing@ccs.ufsc.br. ${ }^{2}$ Faculdade de Saúde Pública, Universidade de São Paulo.

\begin{abstract}
This study sought to describe the main characteristics of epidemiological studies that investigated the association between socioeconomic conditions and head and neck cancer. Research was carried out on the Medline (International Science Literature), Lilacs (Latin American and Caribbean Health Sciences Literature) and Scielo (Scientific Electronic Library Online) databases, as well as the references cited in the articles obtained through the primary search of the aforementioned databases. The publication period considered included 38 years (1970-2007) and the analysis was restricted to articles in Spanish, English or Portuguese. Twenty-five studies were selected, 15 that outlined case-control, four ecological and six that combined information from of ficial databases, such as censuses and cancer or death records. Most cases reported an association between lower socioeconomic conditions and head and neck cancer. The most used indexes were occupation and education. Few studies investigated medication, aiming to point out which proximal factors operate in the investigated association. Additional studies, with uniform criteria to effect the adjustments in the regression models and sufficient samples, are required to inquire this dimension.
\end{abstract}

Key words Head and neck neoplasms, Health inequalities, Socioeconomic factors
Resumo O presente estudo teve como objetivo descrever as principais características dos estudos epidemiológicos que investigaram a associação entre condições socioeconômicas e câncer de cabeça e pescoço. Foram pesquisadas as bases de dados Medline (Literatura Internacional em Ciências), Lilacs (Literatura Latino-Americana e do Caribe em Ciências da Saúde) e Scielo (Scientific Electronic Library Online), além de referências citadas nos artigos obtidos a partir da busca primária nessas bases. O período de publicação considerado englobou 38 anos (1970-2007) e a análise restringiuse aos artigos em espanhol, inglês ou português. Foram selecionados 25 estudos, $15 \mathrm{com}$ delineamento caso-controle, quatro ecológicos e seis que mesclaram informações de bases de dados oficiais, como censos e registros de câncer ou de óbitos. A maior parte das pesquisas reportou associação entre piores condições socioeconômicas e câncer de cabeça e pescoço. Os indicadores mais empregados foram a ocupação e a escolaridade. Poucos estudos investigaram mediação, procurando evidenciar quais os fatores proximais operam na associação investigada. Pesquisas adicionais, com critérios uniformes para proceder aos ajustes nos modelos de regressão e amostra suficiente, são necessárias para investigar essa dimensão.

Palavras-chave Neoplasias de cabeça e pescoço, Desigualdades em saúde, Fatores socioeconômicos 


\section{Introdução}

Em razão de sua significativa incidência, prevalência e mortalidade, o câncer de cabeça e pescoço é considerado um dos principais tumores no Brasil e no mundo. Estima-se que, em 2002, havia 1,6 milhão de pessoas com câncer de cabeça e pescoço em todo o mundo. No mesmo ano, surgiram em torno de 645 mil novos casos da doença e ocorreram cerca de 350 mil mortes. No Brasil, em 2006, estima-se que apenas a cavidade bucal tenha sido responsável por 13.470 novos casos de câncer ${ }^{1}$. Além da significativa incidência, mortalidade e custo econômico, o câncer de cabeça e pescoço origina impactos negativos, e muitas vezes devastadores, na qualidade de vida dos pacientes. Tratamentos mutiladores podem se associar a disfonia, disfagia e desfigurações faciais relevantes, com perda funcional e repercussão no relacionamento social ${ }^{2-4}$.

Os principais fatores associados ao câncer de cabeça e pescoço são o consumo de tabaco e de bebidas alcoólicas, havendo efeito sinérgico pelo consumo frequente de ambos os produtos. A consistência e a força dessas associações, bem como o aumento cumulativo do risco de acordo com o tempo de duração do hábito e a dose de consumo, foram demonstradas em numerosos estudos de coorte e caso-controle ${ }^{5,6}$. Uma alimentação pobre em vegetais e frutas ${ }^{7,8} \mathrm{e}$ fatores ocupacionais - como o trabalho em ambiente externo (com prolongada exposição solar), exposição ao asbesto e a outras substâncias químicas também vêm sendo associados ao câncer de cabeça e pescoço $0^{6,9,10}$.

Estima-se que até $80 \%$ dos casos dessa neoplasia poderiam ser evitados diminuindo-se a exposição das pessoas ao longo de suas vidas aos fatores de risco já identificados ${ }^{11}$. Os conhecimentos gerados ao longo de décadas de estudos, no entanto, não têm impedido que haja distribuição desigual na incidência e na mortalidade por esse tumor de acordo com os estratos sociais das populações ${ }^{12-14}$. Há na literatura uma diversidade de estudos que investigaram a associação entre condições socioeconômicas e câncer de cabeça e pescoço, sendo utilizadas diferentes variáveis exploratórias, diversos ajustes possíveis e sítios anatômicos analisados. Assim, o presente estudo objetivou sistematizar os estudos que investigaram a associação entre condições socioeconômicas e câncer de cabeça e pescoço, detalhando os estudos, país, ano, variáveis e ajustes empregados e as direções das associações identificadas.

\section{Métodos}

A revisão sistemática da literatura foi realizada no primeiro semestre de 2007 a partir das bases de dados Medline (Literatura Internacional em Ciências), Lilacs (Literatura Latino-Americana e do Caribe em Ciências da Saúde) e Scielo (Scientific Electronic Library Online). Além disso, foram selecionadas referências citadas nos artigos obtidos a partir da busca primária nessas bases. Os descritores empregados para a seleção dos estudos, obtidos junto ao DeCS/MeSH (Descritores em Ciências da Saúde), foram: mouth/oral neoplasm/cancer, pharyngeal/pharynx neoplasm/ cancer, laryngeal/larynx neoplasm/cancer, head and neck neoplasm/cancer, upper aerodigestive tract neoplasm e social class, socioeconomic status, socioeconomic factor e inequality.

O período de publicação considerado englobou 38 anos (1970-2007) e a análise restringiu-se aos artigos redigidos em espanhol, inglês ou português. Foram selecionados estudos epidemiológicos analíticos, sendo excluídos relatos de caso, estudos descritivos e outras revisões de literatura. No resumo, deveria estar claro dentre os objetivos o intuito de se testar a associação entre desigualdades socioeconômicas e incidência ou mortalidade por câncer de cabeça e pescoço ou por tumores da boca, faringe e laringe em separado.

Para cada artigo foi preenchida uma ficha a fim de sistematizar as informações metodológicas e os principais resultados dos estudos. A ficha contemplava: (1) o delineamento da pesquisa - foram considerados três grupos de estudo: caso-controle, ecológico e pesquisas que mesclaram bases de dados oficiais (linkage), comumente censos, registros de câncer e de óbitos; (2) país e período em que a pesquisa foi conduzida; (3) população total envolvida no estudo ou unidades de análise (ecológico); (4) localização topográfica do tumor analisado; (5) desfecho investigado (incidência, prevalência ou mortalidade); (6) indicador socioeconômico empregado; (7) direção da associação entre câncer de cabeça e pescoço e condições socioeconômicas; e (8) variáveis utilizadas para ajuste.

\section{Resultados e discussão}

Vinte e cinco artigos atenderam aos critérios de inclusão e compuseram a amostra final analisada. Destes, quinze foram estudos caso-controle e apresentaram o odds ratio como medida de associação para testar as diferenças entre os gru- 
pos socioeconômicos. Seis pesquisas mesclaram bases de dados oficiais (censitárias, de registros de câncer e de óbitos) e apresentaram as diferenças das taxas de mortalidade/incidência ou os riscos relativos segundo os estratos sociais. Por fim, outros quatro estudos foram ecológicos e compararam majoritariamente através de coeficientes de correlação as taxas de incidência ou mortalidade com as condições de vida da população.

Quanto aos estudos caso-controle, pesquisas conduzidas nos EUA, Índia, Itália, França, Canadá, Alemanha, Espanha, Dinamarca, Inglaterra e Brasil apresentaram, em maior proporção, associação entre câncer de cabeça e pescoço e piores condições socioeconômicas (Tabela 1). No grupo de estudos que não identificaram associações estatisticamente significantes, ressaltam-se as amostras reduzidas de casos empregadas em algumas pesquisas. Na maior parte dos estudos, os indicadores socioeconômicos utilizados foram escolaridade e ocupação. A forma de estratificação foi bastante variada e nem sempre foram apresentadas as justificativas dos pontos de corte e as teorias que guiaram os agrupamentos ocupacionais. Outros estudos não relataram a categoria de referência empregada ${ }^{15-17}$. Quanto à escolaridade, Rao e Desai ${ }^{18}$ descreveram, em estudo conduzido na Índia, que as pessoas analfabetas tiveram chance $41 \%$ mais elevada de câncer de língua quando comparadas às alfabetizadas. Tomando o analfabetismo como categoria de exposição, Escribano Uzcudun et al. ${ }^{15}$ relataram, na Espanha, um OR para câncer de faringe de 5,6 em relação às pessoas com ensino básico completo. Maier e Tisch ${ }^{19}$, na Alemanha, identificaram 2,4 vezes mais chances de as pessoas com escolaridade inferior ao ensino secundário apresentarem câncer de laringe que as pessoas com ensino superior ou secundário. Para o mesmo sítio anatômico, Faggiano et al. ${ }^{20}$ descreveram na Itália um OR de 2,23, comparando pessoas com ensino primário e com ensino universitário. Também na Itália, Ferraroni et al. ${ }^{21}$ e Bosetti et al. ${ }^{22}$ relataram, entre 1984 e 1992, maior chance de câncer de boca e faringe para pessoas com menos de sete anos de estudo, em relação a 12 ou mais (OR:2,24; $\left.\mathrm{IC}_{95 \%} 1,44-3,49\right)$. Essa diferença, no entanto, deixou de ser significativa no período 1992-7.

No Brasil, Bouchardy et al. ${ }^{23}$ agruparam os óbitos ocorridos no município de São Paulo entre 1978 e 1982 para estimar, através de estudo caso-controle, a razão de chances de câncer segundo o nível educacional. Considerando os outros cânceres como controles, foi identificada proteção para o câncer de boca e laringe entre as pes- soas com mais de onze anos de estudo em relação às pessoas com menos de um ano de estudo. No sentido contrário, entretanto, foi descrita maior chance para as pessoas com 1-8 anos e 9-11 anos de estudo no caso do câncer de laringe e de 1-8 anos para o câncer de boca. Já os estudos de Greenberg et al. ${ }^{24}$ e Williams e Horm ${ }^{25}$ não relataram desigualdades entre os estratos educacionais para o câncer de cabeça e pescoço na análise bruta e na análise ajustada. Spitz et al. ${ }^{26}$ descreveram maior chance de câncer de glândulas salivares entre as mulheres com maior escolaridade.

Os estudos que utilizaram a ocupação como indicador socioeconômico também identificaram maior razão de chances para os grupos populacionais mais desprivilegiados. Na Alemanha, Maier e Tisch ${ }^{19}$ adotaram como categoria de referência o grupo de pessoas com emprego que exigia curso técnico ou superior e relataram chance 4,2 vezes maior de câncer de laringe para aqueles com empregos sem exigência de habilidades específicas ou trabalhando como aprendizes. No mesmo sentido, na Espanha ${ }^{15}$, na Dinamarca ${ }^{27}$ e no Canadá ${ }^{16}$ piores ocupações foram associadas com maior chance de câncer de boca, faringe e laringe, respectivamente. Na Itália, Faggiano et al. ${ }^{20}$ destacaram maior OR para ocupações manuais e Bosetti et al. ${ }^{22}$ descreveram chance crescente de câncer de laringe para as piores ocupações, registradas segundo o British Registrar General's Social Class ${ }^{28,29}$, a forma de estratificar ocupação mais comumente empregada nos artigos revisados. No entanto, assim como para a escolaridade, Bosetti et al. ${ }^{22}$ relataram que essa associação deixou de ser identificada no período mais recente. Já os estudos de Ferraroni et al. ${ }^{21}$ e Greenberg et al. ${ }^{24}$ não identificaram diferenças entre as ocupações exercidas e a chance de câncer de cabeça e pescoço. Menvielle et al. ${ }^{14}$, em estudo caso-controle na França, investigaram a mediação do consumo de álcool e do tabagismo nas desigualdades socioeconômicas do câncer de hipofaringe e laringe. Os autores relataram que as diferenças entre os estratos sociais na exposição ao tabaco e ao álcool explicaram parcialmente as diferenças de escolaridade e ocupação quanto aos tumores, porém ainda havia efeito residual. No entanto, após ajuste pelas exposições ocupacionais específicas, não houve mais diferenças significativas.

No que diz respeito aos estudos ecológicos (Tabela 2), Hobdell et al..$^{30}$ analisaram as bases de dados da International Agency for Research on Cancer (IARC) e da Organização Mundial da Saúde (OMS) para testar as associações entre diversos desfechos em saúde bucal e indicadores 


\begin{tabular}{|c|c|c|c|c|}
\hline $\begin{array}{l}\text { Tabela 1. Estu } \\
1970-2007 .\end{array}$ & los caso-contr & ole sobre desigualc & dades socioeconômicas e cânc & er de cabeça e pescoço, \\
\hline Local, ano & $\begin{array}{c}\text { Sítio } \\
\text { anatômico }\end{array}$ & $\begin{array}{l}\text { Total de casos- } \\
\text { controles }\end{array}$ & Associações testadas & Ajustes \\
\hline $\begin{array}{l}\text { EUA, } \\
1984-5^{24}\end{array}$ & $\begin{array}{l}\text { Boca e } \\
\text { faringe }\end{array}$ & $762 / 837$ & $\begin{array}{l}\text { Escolaridade }(\cdot) \text {, ocupação } \\
(\bullet) \text { e proporção de anos } \\
\text { trabalhados }(+)\end{array}$ & $\begin{array}{l}\text { Tabagismo, consumo de álcool, idade, } \\
\text { raça, estado civil, área geográfica, perda } \\
\text { dentária, problemas com prótese e entre } \\
\text { as variáveis socioeconômicas }\end{array}$ \\
\hline $\begin{array}{l}\text { Índia, } \\
1980-4^{18}\end{array}$ & Língua & $637 / 635$ & Escolaridade $(+)$ & Idade e região de residência \\
\hline $\begin{array}{l}\text { Itália, } \\
1984-2^{22}\end{array}$ & $\begin{array}{l}\text { Boca e } \\
\text { faringe }\end{array}$ & $528 / 5.408$ & $\begin{array}{l}\text { Escolaridade }(+) \\
\text { e ocupação }(+)\end{array}$ & $\begin{array}{l}\text { Tabagismo, consumo de álcool, idade, } \\
\text { sexo e centro de estudo }\end{array}$ \\
\hline $\begin{array}{l}\text { Itália, } \\
1992-7^{22}\end{array}$ & $\begin{array}{l}\text { Boca e } \\
\text { faringe }\end{array}$ & $528 / 5.408$ & $\begin{array}{l}\text { Escolaridade }(.) \\
\text { e ocupação }(.)\end{array}$ & $\begin{array}{l}\text { Tabagismo, consumo de álcool, idade, } \\
\text { sexo e centro de estudo }\end{array}$ \\
\hline $\begin{array}{l}\text { França, } \\
1989-91^{14}\end{array}$ & $\begin{array}{l}\text { Hipofaringe } \\
\text { e laringe }\end{array}$ & $504 / 242$ & $\begin{array}{l}\text { Escolaridade (+) e ocupação } \\
(+) \text { no modelo ajustado, e } \\
\text { (.) no modelo ajustados } \\
\text { para ambos. }\end{array}$ & $\begin{array}{l}\text { Tabagismo, consumo de álcool, idade e } \\
\text { entre as variáveis socioeconômicas }\end{array}$ \\
\hline $\begin{array}{l}\text { Canadá, } \\
1977-80^{16}\end{array}$ & $\begin{array}{l}\text { Boca, } \\
\text { hipofaringe, } \\
\text { orofaringe e } \\
\text { laringe }\end{array}$ & $374 / 374$ & Ocupação $(+)$ & $\begin{array}{l}\text { Tabagismo, consumo de álcool, sexo, } \\
\text { idade, estado civil, consultas } \\
\text { odontológicas e história de tuberculose }\end{array}$ \\
\hline $\begin{array}{l}\text { Itália, } \\
1980-3^{17}\end{array}$ & Lábio & $53 / 106$ & $\begin{array}{l}\text { Índice composto } \\
\text { (escolaridade, ocupação, } \\
\text { local de moradia e higiene } \\
\text { pessoal) }(+)\end{array}$ & $\begin{array}{l}\text { Tabagismo, idade e município de } \\
\text { residência }\end{array}$ \\
\hline $\begin{array}{l}\text { Alemanha, } \\
1988-9^{19}\end{array}$ & Laringe & $164 / 656$ & $\begin{array}{l}\text { Escolaridade }(+) \\
\text { e ocupação }(+)\end{array}$ & Tabagismo e consumo de álcool \\
\hline $\begin{array}{l}\text { Espanha, } \\
1990-5^{15}\end{array}$ & Faringe & $232 / 232$ & $\begin{array}{l}\text { Escolaridade }(+) \text {, ocupação } \\
(+) \text { e não descrito }(+)\end{array}$ & $\begin{array}{l}\text { Tabagismo, consumo de álcool, sexo e } \\
\text { idade }\end{array}$ \\
\hline $\begin{array}{l}\text { EUA, } \\
1985-9^{26}\end{array}$ & $\begin{array}{l}\text { Glândulas } \\
\text { salivares }\end{array}$ & $64 / 128$ & $\begin{array}{l}\text { Escolaridade }(-) \text { no sexo } \\
\text { feminino e }(\bullet) \text { no sexo } \\
\text { masculino }\end{array}$ & $\begin{array}{l}\text { Consumo de álcool, radioterapia } \\
\text { anterior, uso de tinta para pintar o } \\
\text { cabelo e uso de enxaguatório bucal }\end{array}$ \\
\hline $\begin{array}{l}\text { Dinamarca, } \\
1980-2^{27}\end{array}$ & Laringe & $326 / 1.134$ & Ocupação $(+)$ & Tabagismo, consumo de álcool e idade \\
\hline $\begin{array}{l}\text { Inglaterra, } \\
1998-2000^{39}\end{array}$ & Boca & $100 / 100$ & $\begin{array}{l}\text { Tempo empregado }(+) \text { no } \\
\text { modelo bruto e }(\bullet) \text { no } \\
\text { modelo ajustado }\end{array}$ & $\begin{array}{l}\text { Tabagismo, consumo de álcool, sexo e } \\
\text { idade }\end{array}$ \\
\hline $\begin{array}{l}\text { Itália, } \\
1983-8^{21}\end{array}$ & $\begin{array}{l}\text { Boca e } \\
\text { faringe }\end{array}$ & $50 / 1944$ & $\begin{array}{l}\text { Ocupação }(+) \text { no modelo } \\
\text { bruto e }(\bullet) \text { no modelo } \\
\text { ajustado; educação }(+) \text { em } \\
\text { ambos }\end{array}$ & $\begin{array}{l}\text { Tabagismo, consumo de álcool, sexo, } \\
\text { idade, estado civil e consumo de café }\end{array}$ \\
\hline $\begin{array}{l}\text { EUA, } \\
1969-71^{25}\end{array}$ & $\begin{array}{l}\text { Lábio e } \\
\text { língua, } \\
\text { gengiva e } \\
\text { cavidade } \\
\text { bucal, } \\
\text { glândulas } \\
\text { salivares, } \\
\text { faringe e } \\
\text { laringe }\end{array}$ & $\begin{array}{l}74,16,53,47, \\
99 \text { (respectivam- } \\
\text { ente, para cada } \\
\text { sítio anatômi- } \\
\text { co) } / 4.976\end{array}$ & Escolaridade $(\bullet)$ e renda $(\bullet)$ & Tabagismo, idade e cor da pele \\
\hline $\begin{array}{l}\text { Itália, } \\
1985-7^{20}\end{array}$ & Laringe & $225 / 16.913$ & $\begin{array}{l}\text { Escolaridade }(+), \text { posse de } \\
\text { casa }(+) \text { e ocupação }(+)\end{array}$ & $\begin{array}{l}\text { Idade, região de residência e entre as } \\
\text { variáveis socioeconômicas }\end{array}$ \\
\hline $\begin{array}{l}\text { Brasil, } \\
1978-2^{23}\end{array}$ & $\begin{array}{l}\text { Boca, } \\
\text { faringe e } \\
\text { laringe }\end{array}$ & $\begin{array}{l}392,277 \text { e } 588 \text { / } \\
8.787,8.902 \text { e } \\
8.591 \\
\text { (respectivamente, } \\
\text { para cada sítio } \\
\text { anatômico) }\end{array}$ & $\begin{array}{l}\text { Escolaridade: boca }(.) ; \\
\text { faringe }(+) \text {; laringe }(+) \\
\text { (consideradas as categorias } \\
\text { extremas); laringe }(-) \\
\text { (consideradas as categorias } \\
\text { intermediárias) }\end{array}$ & Idade, estado civil e país de nascimento \\
\hline
\end{tabular}

(•): associação estatisticamente não significante; (+): associação entre pior condição socioeconômica e maior chance/risco do tumor; (-): associação entre melhor condição socioeconômica e maior chance/risco do tumor. 
socioeconômicos. Em relação ao câncer de boca, foram estudadas a incidência e a mortalidade no sexo masculino e feminino em até 164 países (número que variou de acordo com a disponibilidade dos dados). Foi encontrada maior mortalidade por câncer de boca entre os homens nos países com menor índice de desenvolvimento humano (IDH), média de anos de estudo e produto interno bruto (PIB). No sexo feminino, os mesmos fatores foram identificados como significantes; foi também indicada maior mortalidade nos países com maior taxa de crescimento populacional urbano, mortalidade infantil e mortes de crianças com menos de cinco anos de idade. Quanto à incidência, nenhuma associação foi estabelecida no sexo masculino; mas, para mulheres, foi encontrada maior taxa de casos novos nos países com menor IDH, média de anos de estudo e PIB, e naqueles com maior taxa de crescimento populacional urbano.

$\mathrm{Na}$ Inglaterra, O'Hanlon et al. ${ }^{31}$ investigaram a incidência de câncer de língua e de boca entre 1976-85 e 1986-91 para ambos os sexos e relataram maiores taxas nas regiões mais desprivilegiadas do nordeste do país, com exceção do câncer de língua para mulheres em ambos os períodos e de boca no quinquênio mais recente. No Brasil, Franco et al. ${ }^{32}$ consideraram os estados brasileiros como unidades de análise e, com base nos dados do Registro Nacional de Patologia Tumoral, testaram a correlação entre indicadores socioeconômicos e a frequência relativa do câncer de boca e de laringe. Entre os homens, não houve significância estatística, porém entre as mulheres foi descrita associação positiva do câncer de boca com a mortalidade infantil e negativa do câncer de laringe com praticamente todos os indicadores socioeconômicos avaliados. Antunes et al. ${ }^{13}$ empregaram como unidades de análise espaços geográficos mais homogêneos: as unidades administrativas do município de São Paulo. Os autores identificaram que regiões da cidade onde havia maior pobreza, desigualdade na distribuição de renda, desemprego, analfabetismo, menor instrução e baixa renda registraram maior mortalidade por câncer de boca.

Outros estudos valeram-se da reunião de informações oriundas de fontes diferentes, para estimar taxas de mortalidade e de incidência segundo grupos socioeconômicos (Tabela 3). Levi et al. ${ }^{33}$ mesclaram informações de um registro de câncer da região de Vaud, na Suíça, com os registros nacionais de óbitos e verificaram que a mortalidade proporcional por câncer de boca, faringe, laringe e esôfago foi maior entre 1977 e 1984 nos homens com piores ocupações. A renda também se mostrou importante descritora das desigualdades na incidência de boca/gengiva e larin$\mathrm{ge}^{34}$. Com os dados do Third National Cancer Survey conduzido nos EUA e do censo nacional, foi observado que surgiram no país entre 1969 e 1971 mais casos novos por 100.000 habitantes entre os mais pobres em relação aos mais ricos (exceto para laringe no sexo feminino). Na Itália, Faggiano et al ${ }^{35}$ mesclaram as informações oriundas do censo de 1981 e do registro nacional de óbitos para calcular as taxas de mortalidade por câncer de acordo com os níveis educacionais. Pessoas analfabetas, apenas alfabetizadas e com até quatro anos de estudo apresentaram maior chance de mortalidade para os cânceres de lábio, boca e faringe em relação aos universitários. Maior exposição aos fatores de risco e menor acesso aos serviços de saúde por parte dos menos escolarizados, segundo os autores, devem ter sido os principais meios que determinaram as desigualdades observadas.

Na Finlândia, Raitiola e Pukander ${ }^{36}$ descreveram risco relativo para o câncer de laringe 4,3 vezes maior entre os profissionais não especializados em relação aos gerentes e altos administradores. Além disso, nas ocupações intermediárias, como agricultores, e nas que exigiam trabalho técnico ou especializado foi observado maior risco da neoplasia. O maior consumo de tabaco entre os grupos socioeconômicos mais desprivilegiados no país foi apontado como principal responsável pela desigualdade. Em outro país escandinavo, a Dinamarca, foi descrito maior risco de câncer de laringe entre trabalhadores assalariados com baixa escolaridade quando comparados com os de alta escolaridade ${ }^{37}$. No entanto, a pesquisa não observou diferenças entre as demais ocupações investigadas. Na Nova Zelândia, Pearce e Howard ${ }^{38}$ empregaram a British Registrar General's Social Class para classificar as ocupações e testar as desigualdades socioeconômicas na mortalidade por câncer no país. Observou-se que nos estratos com melhores ocupações a mortalidade por câncer de laringe foi menor. As taxas mais elevadas de mortalidade foram relatadas para as ocupações com trabalho semiespecializado e não especializado.

Dentre as limitações metodológicas identificadas a partir do presente estudo, estão a pequena amostra empregada em diversas pesquisas, a extensa pluralidade de ajustes/controles realizados e a não apresentação de categorias de referência ou detalhes sobre as formas como as variáveis socioeconômicas foram categorizadas. Como 
exemplo, Williams e Horm ${ }^{25}$ estratificaram as suas análises por sexo e localização topográfica do tumor e obtiveram apenas vinte mulheres com câncer de lábio e língua, 16 casos em cada sexo de câncer de glândulas salivares e 47 homens com câncer de faringe. Neste estudo, a não apresentação dos intervalos de confiança também prejudicou melhor avaliação das medidas relatadas. No estudo de Ferraroni et al. ${ }^{21}$, a mesma limitação foi verificada, havendo apenas cinquenta pacientes com câncer de boca e faringe, sendo que destes, por falta de informações, onze não puderam ser classificados dentro das categorias de ocupação.

A maior parte dos estudos caso-controle incorporou em suas análises o ajuste das medidas de efeito pela idade, tabagismo e consumo de álcool; no entanto, algumas pesquisas não realizaram esse procedimento. Além disso, em poucas investigações foram apresentadas as medidas de efeito das categorias socioeconômicas antes e após a incorporação das variáveis de controle no modelo. Tal conduta permitiria estimar quais fatores mediam a ação das condições socioeconômicas na ocorrência do câncer, porém identificou-se que essa dimensão ainda está pouco explorada nas investigações epidemiológicas sobre o tema.

A associação direta entre piores condições socioeconômicas e câncer de cabeça e pescoço destacada no presente estudo, através da revisão de pesquisas conduzidas em diversos países ao longo de quase três décadas, impõe à sociedade a discussão sobre medidas equitativas a serem encaminhadas a fim não apenas de diminuir a incidência e a mortalidade por esse tumor, mas também de eliminar a desigual distribuição da doença entre os estratos sociais.

Tabela 2. Estudos ecológicos sobre desigualdades socioeconômicas e câncer de cabeça e pescoço, 1970-2007.

\begin{tabular}{|c|c|c|c|c|}
\hline $\begin{array}{l}\text { Local, } \\
\text { ano }\end{array}$ & $\begin{array}{c}\text { Sítio } \\
\text { anatômico }\end{array}$ & Desfecho & Indicadores & Unidade de análise \\
\hline \multirow[t]{2}{*}{$\begin{array}{l}\text { Brasil, } \\
1976-80^{32}\end{array}$} & Boca & $\begin{array}{l}\text { Frequência } \\
\text { relativa }\end{array}$ & $\begin{array}{l}\text { Casas com: esgoto }(\bullet) \text {, água encanada }(\bullet) \text {, } \\
\text { telefone }(\bullet) \text {, geladeira }(\bullet) \text {, televisão }(\bullet) \text {, } \\
\text { automóvel }(\bullet) \text {, aglomeração }(\bullet) \text {, renda } \\
\text { inferior a cinco salários mínimos }(\bullet) \text { e } \\
\text { mortalidade infantil }(+)^{*}\end{array}$ & $\begin{array}{l}23 \text { unidades federativas do } \\
\text { Brasil }\end{array}$ \\
\hline & Laringe & $\begin{array}{l}\text { Frequência } \\
\text { relativa }\end{array}$ & $\begin{array}{l}\text { Casas com geladeira }(\bullet) \text {, aglomeração }(\bullet) \text {; } \\
\text { renda inferior a cinco salários mínimos }(\bullet) \text {; } \\
\text { mortalidade infantil }(\bullet) \text {, esgoto }(-)^{*} \text {, água } \\
\text { encanada }(-)^{*} \text {, televisão }(-)^{*} \text {, automóvel }(-)^{*} \text { e } \\
\text { telefone }(-)^{*}\end{array}$ & \\
\hline \multirow[t]{2}{*}{$\begin{array}{l}\text { Diversos } \\
\text { países, } \\
1990^{30}\end{array}$} & $\begin{array}{l}\text { Boca e } \\
\text { faringe }\end{array}$ & Mortalidade & $\begin{array}{l}\text { IDH }(+) \text {, anos de estudo }(+) \text {, taxa de } \\
\text { crescimento populacional urbano }(+)^{*} \text {, PIB } \\
(+) \text {, mortalidade infantil }(+)^{*} \text {, mortalidade de } \\
<5 \text { anos }(+)^{*} \text {, Gini }(\cdot)\end{array}$ & $\begin{array}{l}\text { Entre } 102 \text { e } 164 \text { países do } \\
\text { mundo, variando de } \\
\text { acordo com o indicador }\end{array}$ \\
\hline & & Incidência & $\begin{array}{l}\text { IDH }(+)^{*} \text {, anos de estudo }(+)^{*} \text {, taxa de } \\
\text { crescimento populacional urbano }(+)^{*} \text {, PIB } \\
(+)^{*} \text {, mortalidade infantil }(\bullet) \text {, mortalidade de } \\
<5 \text { anos }(\bullet) \text {, Gini }(\bullet)\end{array}$ & \\
\hline \multirow[t]{2}{*}{$\begin{array}{l}\text { Inglaterra, } \\
1976-85^{31}\end{array}$} & Língua & Incidência & $\begin{array}{l}\text { Privação material (propriedade de moradia e } \\
\text { de carro, aglomeração e desemprego) }(+)^{* *}\end{array}$ & $\begin{array}{l}678 \text { distritos regionais no } \\
\text { nordeste da Inglaterra }\end{array}$ \\
\hline & Boca & Incidência & $\begin{array}{l}\text { Privação material (propriedade de moradia e } \\
\text { de carro, aglomeração e desemprego) }(+)\end{array}$ & \\
\hline \multirow[t]{2}{*}{$\begin{array}{l}\text { Inglaterra, } \\
1986-91^{31}\end{array}$} & Língua & Incidência & $\begin{array}{l}\text { Privação material (propriedade de moradia e } \\
\text { de carro, aglomeração e desemprego) }(+)^{* *}\end{array}$ & $\begin{array}{l}678 \text { distritos regionais no } \\
\text { nordeste da Inglaterra }\end{array}$ \\
\hline & Boca & Incidência & $\begin{array}{l}\text { Privação material (propriedade de moradia e } \\
\text { de carro, aglomeração e desemprego) }(+)^{* *}\end{array}$ & \\
\hline $\begin{array}{l}\text { Brasil, } \\
1980-98^{13}\end{array}$ & Boca & Mortalidade & $\begin{array}{l}\text { Renda familiar }(+) \text {, Gini }(+) \text {, taxa de } \\
\text { desemprego }(+) \text {, aglomeração }(+) \text {, } \\
\text { analfabetismo }(+) \text {, ensino médio concluído } \\
(+) \text {, anos de estudo }(+)\end{array}$ & $\begin{array}{l}97 \text { regiões administrativas } \\
\text { do município de São Paulo }\end{array}$ \\
\hline
\end{tabular}

(•): associação estatisticamente não significante; (+): associação entre pior condição socioeconômica e maior chance/risco do tumor; (-): associação entre melhor condição socioeconômica e maior chance/risco do tumor; ${ }^{*}$ : sexo feminino; "*: sexo masculino. 
Tabela 3. Estudos com junção de diferentes bases de dados sobre desigualdades socioeconômicas e câncer de cabeça e pescoço, 1970-2007.

\begin{tabular}{|c|c|c|c|c|}
\hline $\begin{array}{l}\text { Local, } \\
\text { ano }\end{array}$ & $\begin{array}{l}\text { Localização } \\
\text { topográfica }\end{array}$ & População & Indicadores & Medidas e ajustes \\
\hline $\begin{array}{l}\text { Suíça, } \\
1977-84^{33}\end{array}$ & $\begin{array}{l}\text { Boca, faringe, } \\
\text { laringe e } \\
\text { esôfago }\end{array}$ & $\begin{array}{l}530 \text { mil pessoas; } 409 \text { casos } \\
\text { homens e } 63 \text { casos mulheres }\end{array}$ & Ocupação $(+)^{* *}$ & $\begin{array}{l}\text { Taxa de mortalidade } \\
\text { proporcional ajustada pela idade }\end{array}$ \\
\hline \multirow[t]{2}{*}{$\begin{array}{l}\text { EUA, } \\
1969-71^{34}\end{array}$} & Boca e gengiva & $\begin{array}{l}21 \text { milhões de pessoas; } 843 \text { casos } \\
\text { homens e } 450 \text { casos mulheres }\end{array}$ & Renda (+) & \multirow[t]{2}{*}{$\begin{array}{l}\text { Taxa de incidência por } 100.000 \\
\text { pessoas }\end{array}$} \\
\hline & Laringe & $\begin{array}{l}1.825 \text { casos homens e } 251 \text { casos } \\
\text { mulheres }\end{array}$ & Renda $(+)^{* *}$ & \\
\hline \multirow{3}{*}{$\begin{array}{l}\text { Finlândia, } \\
1962-91^{36} \\
\text { Itália, } \\
1981-2^{35}\end{array}$} & Laringe & $\begin{array}{l}400 \text { mil pessoas; } 302 \text { casos } \\
\text { homens }\end{array}$ & Ocupação (+) & Risco relativo ajustado pela idade \\
\hline & $\begin{array}{l}\text { Lábio, boca e } \\
\text { faringe }\end{array}$ & $\begin{array}{l}\text { 8.903.526 homens (pessoas- } \\
\text { ano); } 725 \text { casos homens. } \\
\text { 9.441.897 mulheres (pessoas } \\
\text { ano); } 107 \text { casos mulheres }\end{array}$ & Escolaridade $(+)^{* *}$ & \multirow[t]{2}{*}{$\begin{array}{l}\text { Razão de taxas de mortalidade } \\
\text { ajustada por idade e região de } \\
\text { residência }\end{array}$} \\
\hline & Laringe & 817 casos homens & Escolaridade $(+)$ & \\
\hline $\begin{array}{l}\text { Nova } \\
\text { Zelândia, } \\
1974-8^{38}\end{array}$ & Cavidade bucal & $\begin{array}{l}\text { População total não descrita; } \\
5.356 \text { óbitos por todos os tipos } \\
\text { de câncer; Casos de cavidade } \\
\text { bucal não descritos }\end{array}$ & Ocupação (+) & $\begin{array}{l}\text { Taxa de mortalidade por } \\
100.000 \text { pessoas-ano. }\end{array}$ \\
\hline $\begin{array}{l}\text { Dinamarca, } \\
1970-80^{37}\end{array}$ & $\begin{array}{l}\text { Laringe } \\
\text { Laringe }\end{array}$ & $\begin{array}{l}\text { 9.063.015 homens (pessoas- } \\
\text { ano); } 1.226 \text { homens casos; } \\
\text { 9.632.440 mulheres (pessoas- } \\
\text { ano); } 209 \text { mulheres casos }\end{array}$ & $\begin{array}{l}\text { Ocupação }(+) \\
\text { Ocupação }(+)^{* *}\end{array}$ & $\begin{array}{l}\text { Foi realizada regressão de } \\
\text { Poisson e os parâmetros } \\
\text { interpretados como risco relativo }\end{array}$ \\
\hline
\end{tabular}

(•): associação estatisticamente não significante; (+): associação entre pior condição socioeconômica e maior chance/risco do tumor;

(-): associação entre melhor condição socioeconômica e maior chance/risco do tumor; ${ }^{* *}$ : sexo masculino.

\section{Colaboradores}

AF Boing conduziu a seleção e o fichamento dos artigos e propôs a primeira versão do manuscrito; JLF Antunes orientou a revisão e contribuiu na redação final do texto.

\section{Referências}

1. Brasil. Ministério da Saúde. Instituto Nacional de Câncer. Estimativa 2006: incidência de câncer no Brasil. Rio de Janeiro: Inca; 2005.

2. Andrade FP, Antunes JL, Durazzo MD. Evaluation of the quality of life of patients with oral cancer in Brazil. Pesqui Odontol Bras 2006; 20(4):290-296.

3. Van Cann EM, Dom M, Koole R, Merkx MA, Stoelinga PJ. Health related quality of life after mandibular resection for oral and oropharyngeal squamous cell carcinoma. Oral Oncol 2005; 41(7):687-693.

4. Shepherd KL, Fisher SE. Prospective evaluation of quality of life in patients with oral and oropharyngeal cancer: from diagnosis to three months posttreatment. Oral Oncol 2004; 40(7):751-757.

5. Austin DF. Larynx. In: Schottenfeld D, Fraumeni Jr JF, editors. Cancer epidemiology and prevention. New York: Oxford; 1996. p. 179-193.

6. Mahboubi E, Sayed GM. Oral cavity and pharynx. In: Schottenfeld D, Fraumeni Jr. JF, editors. Cancer epidemiology and prevention. New York: Oxford; 1996. p. 583-595.

7. Zain RB. Cultural and dietary risk factors of oral cancer and precancer: a brief overview. Oral Oncol 2001; 37(3):205-210. 
8. Key TJ, Allen NE, Spencer EA, Travis RC. The effect of diet on risk of cancer. Lancet 2002; 360 (9336):861-868

9. Kogevinas M, Sala M, Boffetta P, Kazerouni N, Kromhout $\mathrm{H}$, Hoar-Zahm S. Cancer risk in the rubber industry: a review of the recent epidemiological evidence. Occup Environ Med 1998; 55(1):1-12.

10. Moore S, Johnson N, Pierce A, Wilson D. The epidemiology of lip cancer: a review of global incidence and aetiology. Oral Dis 1999; 5(3):185-195.

11. Tomatis L, editor. Cancer: causes, ocurrence and control. Lyon: IARC Sci Publ; 1990.

12. Edwards DM, Jones J. Incidence of and survival from upper aerodigestive tract cancers in the UK: the influence of deprivation. Eur J Can 1999; 35(6):968-972.

13. Antunes JL, Biazevic MG, Araújo ME, Tomita NE, Chinellato LE, Narvai PC. Trends and spatial distribution of oral cancer mortality in São Paulo, Brazil, 1980-1998. Oral Oncol 2001; 37(4):345-350.

14. Menvielle G, Luce D, Goldberg P, Leclerc A. Smoking, alcohol drinking, occupational exposures and social inequalities in hypopharyngeal and laryngeal cancer. Int J Epidemiol 2004; 33(4):799-806.

15. Escribano Uzcudun A, Rabanal Retolaza I, Garcia Grande A, Miralles Olivar L, Garcia Garcia A, Gonzalez Baron M, Gavilan Bouzas J. Pharyngeal cancer prevention: evidence from a case-control study involving 232 consecutive patients. J Laryngol Otol 2002; 116(7):523-531.

16. Elwood JM, Pearson JC, Skippen DH, Jackson SM. Alcohol, smoking, social and occupational factors in the aetiology of cancer of the oral cavity, pharynx and larynx. Int J Cancer 1984; 34(5):603-612.

17. Dardanoni L, Gafa L, Paterno R, Pavone G. A casecontrol study on lip cancer risk factors in Ragusa (Sicily). Int J Cancer 1984; 34(3):335-337.

18. Rao DN, Desai PB. Risk assessment of tobacco, alcohol and diet in cancers of base tongue and oral tongue: a case control study. Indian J Cancer 1998; 35(2):65-72.

19. Maier H, Tisch M. Epidemiology of laryngeal cancer: results of the Heidelberg case-control study. Acta Otolaryngol 1997; 527(Suppl.):160-164.

20. Faggiano F, Zanetti R, Costa G. Cancer risk and social inequalities in Italy. J Epidemiol Community Health 1994; 48(5):447-452.

21. Ferraroni M, Negri E, La Vecchia C, D’Avanzo B, Franceschi S. Socioeconomic indicators, tobacco and alcohol in the aetiology of digestive tract neoplasms. Int J Epidemiol 1989; 18(3):556-562.

22. Bosetti C, Franceschi S, Negri E, Talamini R, Tomei F, La Vecchia C. Changing socioeconomic correlates for cancers of the upper digestive tract. Ann Oncol 2001; 12(3):327-330.

23. Bouchardy C, Parkin DM, Khlat M, Mirra AP, Kogevinas M, Lima FD, Ferreira CE. Education and mortality from cancer in São Paulo, Brazil. Ann Epidemiol 1993; 3(1):64-70.

24. Greenberg RS, Haber MJ, Clark WS, Brockman JE, Liff JM, Schoenberg JB, Austin DF, Preston-Martin $S$, Stemhagen A, Winn DM. The relation of socioeconomic status to oral and pharyngeal cancer. Epidemiology 1991; 2(3):194-200.
25. Williams RR, Horm JW. Association of cancer sites with tobacco and alcohol consumption and socioeconomic status of patients: interview study from the Third National Cancer Survey. J Natl Cancer Inst 1977; 58(3):525-547.

26. Spitz MR, Fueger JJ, Goepfert H, Newell GR. Salivary gland cancer: a case-control investigation of risk factors. Arch Otolaryngol Head Neck Surg 1990; 116(10):1163-1196.

27. Olsen J, Sabroe S. Occupational causes of laryngeal cancer. J Epidemiol Community Health 1984; 38(2):117-121

28. Krieger N, Williams DR, Moss NE. Measuring social class in US public health research: concepts, methodology, and guidelines. Annu Rev Public Health 1997; 18:341-378.

29. Szreter SRS. The genesis of the Registrar-Generalls Social Classification of Occupations. Br J Sociol 1984; 35(4):522-546.

30. Hobdell MH, Oliveira ER, Bautista R, Myburgh NG, Lalloo R, Narendran S, Johnson NW. Oral diseases and socio-economic status (SES). Br Dent J 2003; 194(2):91-96.

31. O'Hanlon S, Forster DP, Lowry RJ. Oral cancer in the North-East of England: incidence, mortality trends and the link with material deprivation. Community Dent Oral Epidemiol 1997; 25(5):371-376.

32. Franco EL, Campos Filho N, Villa LL, Torloni H. Correlation patterns of cancer relative frequencies with some socioeconomic and demographic indicators in Brazil: an ecologic study. Int J Cancer 1988; 41(1):24-29.

33. Levi F, Negri E, La Vecchia C, Te VC. Socioeconomic groups and cancer risk at death in the Swiss Canton of Vaud. Int J Epidemiol 1988; 17(4):711-717.

34. Ernster VL, Selvin S, Sacks ST, Merrill DW, Holly EA. Major histologic types of cancers of the gum and mouth, esophagus, larynx, and lung by sex and by income level. J Natl Cancer Inst 1982; 69(4):773-776.

35. Faggiano F, Lemma P, Costa G, Gnavi R, Pagnanelli F. Cancer mortality by educational level in Italy. Cancer Causes Control 1995; 6(4):311-320.

36. Raitiola HS, Pukander JS. Etiological factors of laryngeal cancer. Acta Otolaryngol 1997; 529(Supl.): 215-257.

37. Guenel P, Engholm G, Lynge E. Laryngeal cancer in Denmark: a nationwide longitudinal study based on register linkage data. Br J Ind Med 1990; 47(7):473479.

38. Pearce NE, Howard JK. Occupation, social class and male cancer mortality in New Zealand, 1974-8. Int J Epidemiol 1986; 15(4):456-462.

39. Greenwood M, Thomson PJ, Lowry RJ, Steen IN. Oral cancer: material deprivation, unemployment and risk factor behavior: an initial study. Int J Oral Maxillofac Surg 2003; 32(1):74-77.

Artigo apresentado em 31/10/2007

Aprovado em 14/12/2007

Versão final apresentada em 14/01/2008 